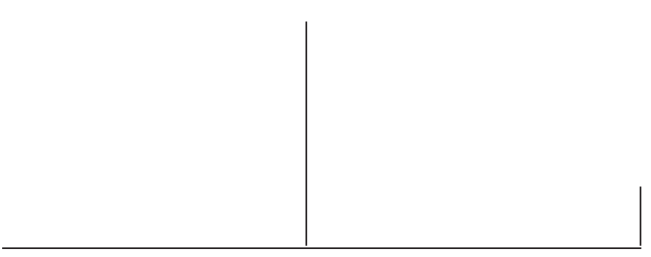

Rev. Latinoam. Psicopat. Fund., V, 4, 85-98

\title{
Georg Cantor: habitar lo eterno
}

Juan Manuel Rodríguez Penagos

Este artículo pone en discusión la relación entre estructura psíquica y la producción lógica del delirio; para ello, parte de un matemático alemán llamado Georg Cantor. Aquí se discute la relación entre la producción matemática y la instalación de su psicosis.

Palabras claves: Infinitos, psicosis, matemáticas, conjuntos 


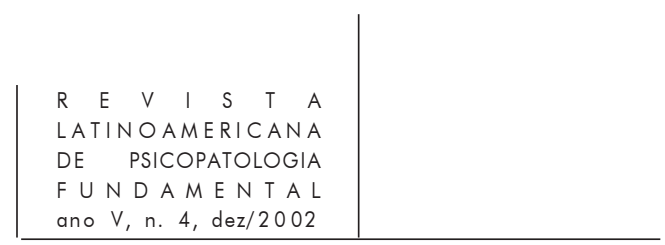

El estilo del deseo es la eternidad.

J. L. Borges

\section{Introducción}

El delirio siempre parece hablar desde las sombras, lugares donde no podemos imaginar espacios; el sujeto se desvanece y aparecen una serie de personajes mitológicos, épicos, dispuestos a salvar al mundo o a acabar con él, estas creaciones animadas no pueden morir, no tienen la vulnerabilidad del cuerpo, son ilimitadas.

Cuando empieza a aparecer el discurso de los pacientes, las tramas comienzan a cambiar, parece organizarse la subjetividad a partir de lo ilimitado; el sueño, por ejemplo, es una dimensión psíquica donde lo inconsciente toma la palabra o, quizá, la imagen; una forma alucinatoria de realización del deseo que parece, en este sentido, ser una ventana a lo infinito de la subjetividad.

El delirio muestra entonces la forma fallida de un sujeto de habitar un espacio, deja de soñar para vivir el deseo alucinado; las batallas épicas y los fantasmas toman cuerpo y espacio; este proceso es entonces también una manera de organizar los infinitos; este es el caso de Georg Cantor.

Partamos de una pregunta: ¿Qué formas adquiere la paternidad? Cada sujeto, desde su diferencia plantea un lugar distinto; la psicosis se caracteriza por la ausencia del Nombredel-Padre, esto no quiere decir que no tenga efectos, sino que es presente por su ausencia, es decir: plantea un lugar de 
destitución. La búsqueda por sostenerse pasa por diferentes territorios, sus primeros estudios de ingeniería, sus interlocutores académicos, su interés por la teología, el lugar de padre de los transfinitos, la creación de su propia familia; los efectos de la paternidad están representados en todos los campos del saber, más aún, definen las formas de simbolizar (o no) de un sujeto; en este sentido, determinan las formas sintomáticas de aprehender del mundo.

Vayamos a nuestro matemático, es importante situar la prehistoria del pensamiento religioso de Cantor desde la historia familiar, especialmente del lado del padre; en la genealogía paterna hubo diversas mitologías religiosas que luchaban entre sí, precisamente luchaban por un lugar de transmisión: pasaban de una religión a otra; el abuelo de Cantor fue judío, quien dio a su hijo una estricta formación como protestante luterano, ahí comenzamos con una escisión significativa, ¿Por qué el abuelo no transmitió a su hijo su religión judía?, ¿Qué pasó con ese Dios del judaísmo?, ¿Por qué se destituyó?; sin embargo, frente a lo absoluto el abuelo decidió trasmitir una religión a la que no pertenecía; es decir, la búsqueda por ubicar lo absoluto comienza generaciones atrás; el padre de Georg, a su vez transmitió a su hijo una estricta formación protestante, interés y respeto que llegaba al miedo y a la sumisión frente a un mundo amenazante que invalidaba al otro.

El padre asimismo transmitió a su hijo una devoción temerosa hacia Dios, la misma divinidad que fundamentaba todos los campos del saber y la actividad humana. Hablar de lo infinito era una de las formas epistemológicas de Dios, es decir del Nombre-del-Padre.

\section{La carta del padre: ¿Un destino?}

Varios autores (Charraud, Dauven) señalan el momento de la confirmación religiosa como crucial en el destino de Cantor, existe una carta que ejemplifica este hecho, especialmente importante pues es una carta escrita por el padre, es decir, la confirmación de un sujeto frente a un padre ilimitado, infinito, la carta lleva impresa una fantasmagoría, un deseo inconsciente del padre: una prueba de destitución; al mismo tiempo se está jugando una entidad ilimitada, una omnipotencia o una impotencia, por tanto, todas estas formas de estructuración psíquica a partir de la paternidad conllevan una lógica del tiempo, de historizarse, un camino.

En esta carta podemos observar la historia de una búsqueda temerosa de Dios desde el abuelo paterno, el cual, en algún momento prefirió trasmitir el Dios Padre distinto al del judaísmo, ¿Por qué?; desde éste momento de la genealogía, 


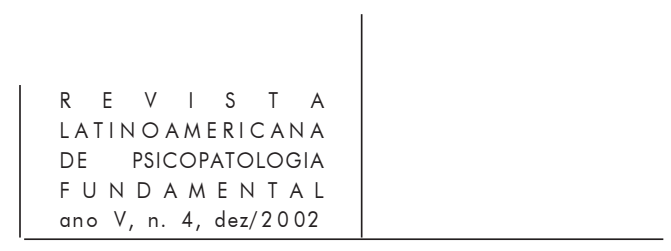

podemos encontrar una falla en este sitio mitológico, vector de estructura; esta carta ritual es un designio paterno, por esta razón decidí transcribirla en su totalidad. Me permití enumerar algunos párrafos para ejemplificar ciertos puntos:

\section{"Muy Querido Georg,}

(1) Por la gracia del todopoderoso, el creador de nuestro universo y el padre de todas las criaturas vivientes, que este día sea de una influencia benigna sobre tu vida futura toda entera.

Puedes tu, de manera constante e incesante, guardar delante de tus ojos las virtuosas resoluciones, y que sin ninguna duda has tomado el día de hoy, en silencio y de manera solemne.

(2) El futuro de una vida y el destino de un individuo, le habitan escondidos en la mas profunda oscuridad. Y es muy cierto que esto es así. Nadie sabe de antemano en que circunstancias de trabajo va a caer por casualidad, contra que calamidades imprevistas e imprevisibles él va a tener que batirse en las diversas situaciones de la vida.

(3) Cuan seguido los individuos más prometedores son derrotados por una dificultad ligera en el ejercicio de su función, con su coraje destrozado, ellos quedan totalmente atrofiados enseguida y también, en el mejor de los casos, ellos serán llamados nada mas unos 'genios arruinados'. De hecho, no es raro que algunos jóvenes lleguen a tal fin, aun aquellos que aparentemente están dotados de características de lo más prometedoras del cuerpo y espíritu, y en donde las perspectivas del futuro cuentan con sus dones, y con buenas relaciones familiares desde su juventud.

(4) Pero les faltaba aquel corazón seguro del cual todo depende. Ahora, mi querido hijo, créeme, yo que soy tu amigo más sincero, el más verdadero, el más experimentado, este corazón fuerte que debe vivir en nosotros, es un espíritu verdaderamente religioso, esto se manifiesta en nosotros como un sentimiento sincero y humilde de la más grande devoción y un reconocimiento hacia Dios; sentimiento a partir del cual, se engrandece y también a la vez inquebrantable, victorioso, en Dios, y que nos guarda y nos mantiene toda nuestra vida entera, en esta silenciosa e indubitable comunión con nuestro padre que esta en los cielos!

(5) Pero con la finalidad de prevenir todas estas pruebas y dificultades que se elevan irresistiblemente contra nosotros, bajo la forma de celos o calumnias de parte de nuestros enemigos secretos o declarados, contra nuestra aspiración ardiente de éxito en el ejercicio de nuestra propia especialidad o actividad, con la finalidad de combatirlos con éxito, nosotros necesitamos sobre todo de adquirir y de apropiarnos de la mayor cantidad posible de conocimientos, de practicas y de técnicas en las diferentes disciplinas de base. Desde nuestros días, esto es de una necesidad absoluta para un hombre ambicioso, que no quiere verse 


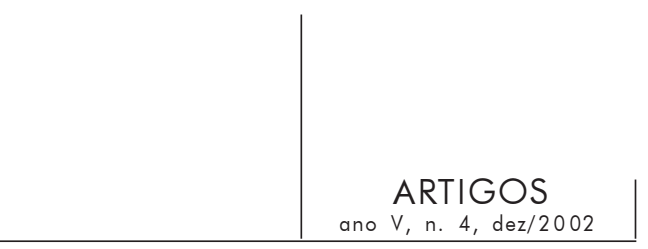

empujado afuera por sus enemigos y obligado a tener un lugar de segundo o de tercer rango.

(6) Tu debes ser plenamente consciente, que el aprendizaje de todo tipo de conocimientos exhaustivos, científicos; así como prácticos, la adquisición perfecta de lenguas extranjeras y de sus literaturas, el desarrollo pluridireccional del espíritu en las numerosas disciplinas humanas; todas esas cosas deben ser adquiridas en el segundo periodo de tu vida, es decir, la juventud, que comienza precisamente aquí; todo esto servirá para equiparte dignamente para el combate a venir; si uno se muestra negligente durante este periodo por un desgaste prematuro de lo mejor de sus fuerzas, de su salud y de sus tiempos, esto es irremplazable e irreparable, perdido para siempre, todo como la inocencia una vez perdida, para siempre y eternamente, irremediablemente perdida.

Yo termino por estas palabras: tu padre, o mas bien tus padres y todos los otros miembros de la familia, de Alemania, Rusia, y de Dinamarca, tienen los ojos sobre ti como primogénito, y esperando de ti nada menos que un Theodore Schaeffer y, si Dios lo quiere, mas tarde, puede ser una estrella brillante en el horizonte de la ciencia." (Charraud, 1994, p. 13, traducción del autor)

En esta carta podemos descubrir algunos rasgos de la trasmisión del Nombre-del-Padre en nuestro matemático. En ella podremos señalar algunas características de este padre. Transmisión paterna en la psicosis muestra fundamentalmente su ausencia. Lugar en el cual el sujeto no podrá referirse para asumir eventualmente un lugar de padre. Este lugar imposible en la psicosis. El fantasma paterno se convirtió en las imágenes del delirio en Cantor. En los siguientes puntos quisiera aportar algunas reflexiones en relación a esta transmisión, pues en ella se inscribe una genealogía, a saber:

(1) El padre no podía hablar en nombre propio sosteniendo este lugar, sólo podía hacerlo través del nombre de dios. No es el padre quien habla sino dios a través de él. Señalando que este día fuera de una "influencia benigna sobre tu vida futura toda entera." Es importante señalar el contexto de la confirmación religiosa. Un contexto ceremonial en acto. Este episodio quizá tendría efectos en la escritura histórica y mitológica de Georg Cantor.

(2) El futuro que describe el padre ya esta escrito y escondido en la oscuridad. Ya esta hecho, sólo tiene que aparecer. Cantor no podría ser un actor de su historia, todo lo que sucedería tendría que aparecer. Además de esta idea sobre el destino, enuncia que nadie puede prever las "... calamidades imprevistas e imprevisibles él va a tener que batirse en las diversas situaciones de la vida...". Entonces además de la idea del destino nadie puede saber a que se enfrentará. Otra vez la idea de que el sujeto esta exento del saber. Solo que ahora la amenaza llega sin avisar desde el afuera. 


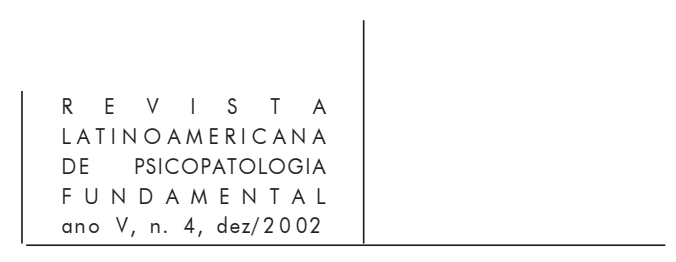

(3) Este trazo del tiempo hecho por el padre, se inscribe como fantasma. Se transmite en este y otras partes de la carta una fantasmagoría casi delirante. El padre vive en su hijo toda esta predestinación. En dos ocasiones el padre alude a la frecuencia con que se producen los "Genios arruinados". Aun los "... mas prometedores de cuerpo y espíritu". Con que podría contar un sujeto así. Ni la genialidad ni los recursos se hacen valer; además este trazo hecho sobre el tiempo aparece también como invalidante; las perspectivas del futuro también engañan. "... y en donde las perspectivas del futuro cuentan con sus dones..." Otra señal de que el sujeto no podría confiar, ni en el tiempo. Así, esta transmisión ejemplifica la escritura del padre en Cantor.

(4) Aquí ubicamos la segunda referencia a dios. La primera ocasión fue al principio de la carta. De nuevo aparece aquí, después de una serie de advertencias. El padre, plantea que la única fortaleza "es un espíritu verdaderamente religioso," fundado en un reconocimiento hacia dios. ¿Es el mismo dios que escribe el destino escondido del que hablaba en párrafos anteriores? El contexto donde incluye el padre a dios es interesante pues propone que la fuerza es igual a la creencia y devoción que se tenga a dios. ¿Qué pasaría si dios no llegase a la cita? ¿Que efectos tendría sobre lo cotidiano? Quizá esta función mitológica fallo en la estructura psíquica de Cantor durante la producción de su obra, teniendo consecuencias necesarias sobre su teoría y los campos epistemológicos en que se fundamento esta búsqueda.

(5) La carta muestra en este segmento la posibilidad de prevenir, cosa que algunos párrafos anteriores habían descartado; también en este párrafo habla de prevenir algo irresistible; en sus propias palabras: "de las pruebas y dificultades que se elevan irresistiblemente contra nosotros". ¿Se puede prevenir algo de lo cual el sujeto no se puede resistir? De nuevo se muestra un sujeto indefenso. Sin embargo sería necesario adquirir todo tipo de conocimientos para enfrentar a sus enemigos. Entonces el saber se plantea como un arma contra los enemigos. La amenaza continua por otros territorios. La ciencia, entonces quizá prometía un refugio para Cantor.

(6) La inconsistencia en el saber tiene un precio muy caro. Este saber que en párrafos anteriores había descartado como útil en la defensa de los embates contra los enemigos. Aun así, después de señalar la importancia del "desarrollo pluridireccional del espíritu" comienza de nuevo la amenaza; esta vez bajo la perdida eterna e irreparable de "... lo mejor de sus fuerzas, de su salud y de sus tiempos....". Nunca es claro el camino que proponen estos pasajes. Después de los recursos viene la destitución. Al final de la carta, dice que sus posibilidades dependen del deseo de Dios. Esta idea multiforme de Dios atraviesa toda la carta bajo la forma de entidad suprasensible que amenaza y controla. 


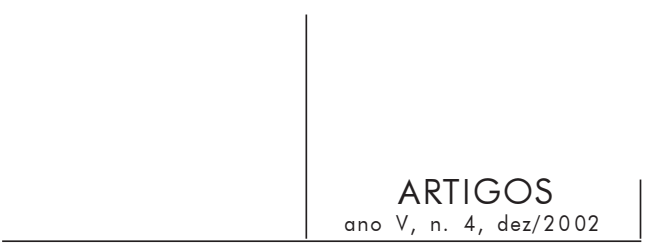

El designio inscrito en esta carta parece ser aceptado en su totalidad. Cantor comienza así la respuesta: "Mi querido padre tu puedes imaginarte cuanto gusto me ha dado tu carta; esta determina mi futuro..." (Ibid., p. 15). Cabe retomar la pregunta que dio titulo a este apartado. ¿Esto significo un destino?

Además del destino inscrito en la carta, la paternidad en Cantor presentó una búsqueda constante: su saber del Dios omnipotente adquirió entonces muchas formas, además de las matemáticas, Cantor buscó sostener en esto un lugar de transmisión, proponiendo en la universidad de Munich sostener un seminario de teología, otra de las formas de construir una silueta de un fantasma divino, ilimitado. Las figuras mitológicas tuvieron una evolución.

Es importante entonces señalar que los fantasmas cantorianos también estaban presentes en la búsqueda teológica y matemática de lo infinito, primero teología, después delirio; primero imaginario, después Real; ${ }^{1}$ la primera forma de contención cantoriana podríamos ubicarla precisamente en la religión, lo cual, como todos los demás intentos, fueron un refugio insuficiente; cabe señalar que además de la teología realizó estudios de astronomía y filosofía. La teoría de los transfinitos era finalmente una lógica para desarrollar ontologicamente su corpus teórico y sus consecuencias.

La carta, en efecto, se convirtió en un destino, trasmitió en ésta toda una trayectoria: la suplantación del padre por una invocación a Dios, habla de un padre ausente, destituido. Su evolución psicopatológica coincide con los perseguidores del padre. Los perseguidores de Cantor fueron precisamente sus anteriores interlocutores académicos; Georg entonces encarna un fantasma paterno y lo lleva hasta su más literal consecuencia: un destino.

\section{El sujeto y su corpus}

La producción matemática guarda una gran relación con el autor, pues desde la teología buscaba ordenar lo infinito, trasmitir una imagen de Dios resignificandolo; la completud tenía mucho sentido en esta búsqueda, por ello, lo infinito venía a representar un campo de estudio esencial. En ese momento existían prejuicios doctrinales sobre la eternidad y la deidad desde hacia 2000 años, el dogma religioso presidía todos los campos de saber humano, el punto de partida fue que solo la inteligencia infinita podía contener estos enigmas, había que transgredir estos dogmas para poder teorizar y, de hecho los efectos fueron establecer otra relación del hombre con Dios, así, el Dios del dogma cayó en 


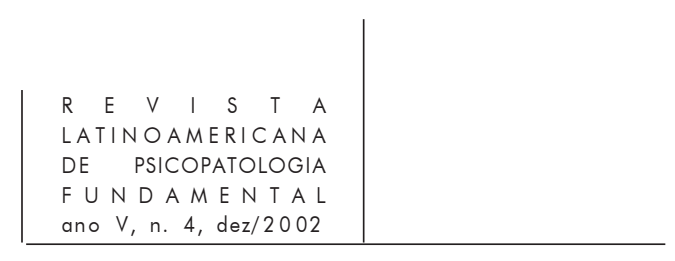

los mismos campos en los que se sostenía con fragilidad, el forzoso lugar de Dios fue retrocediendo a medida que Cantor esgrimía sus argumentos, él tenía una gran obsesión por la consistencia del mundo, ésto lo podemos ver en toda su obra, por ejemplo la paradoja de Aquiles y la tortuga en la que quería explicar la divisibilidad de la línea, es decir, su consistencia; en este campo comienzan las contribuciones revolucionarias de Cantor, es precisamente aquí donde se origina la idea de los números transfinitos, este campo se llama la topología de la línea, y lo desarrolló entre 1879 y 1882, en ese momento la teoría de los conjuntos estaba revolucionando las matemáticas a través de los "conjuntos infinitos y lineales de puntos" (Dauven, 1979), en 1882 presenta los números transfinitos, esto hace a Cantor el fundador de la teoría de los conjuntos. Todo sus desarrollos tenían la obsesión de la completud, del sin-falla.

En los artículos anteriores a los fundamentos de una teoría general de conjuntos, discute el salto entre lo enumerable y lo continuo, que él había demostrado como dos modos de lo infinito; para cerrar la brecha, busca en la topología de la línea, y la estructura de la línea y del espacio (Charraud, 1994, p. 71). La diferencia radical con sus antecesores es que el infinito no es potencial, es actual, presente.

El espacio como modelo matemático tuvo sus efectos a partir de esta primera aportación cantoriana; la topología no sólo afectaba a la matemática, sino también al propio mundo que se vería afectado por este descubrimiento ontológico, el espacio fue una de las principales revoluciones generadas a partir de las aportaciones cantorianas, para quien resolver estos grandes enigmas, propone diferentes modos de abordarlo, por ejemplo, en cuanto a la cuestión de Aquiles y la tortuga, propuso que la continuidad de los espacios diferentes a uno ( $\mathrm{N}$ dimensionales) permite criticar la idea de la continuidad basada en el movimiento; contrario a lo que el sentido común puede decir, dos líneas de diferente longitud tienen el mismo numero de puntos.

Las publicaciones constituyen un reconocimiento social a sus teorías, lo cual permitía en este momento a Cantor sostener el nombre propio; en 1872 escribe su primer articulo en el Matematische Annalen, a los 29 años escribe sobre la teoría de los conjuntos infinitos; publicar ideas tan revolucionarias tenía grandes riesgos, él contaba con aliados como Mittag-Leffer, editor de la revista Acta Matemática; así, para los umbrales del siglo XX, comenzó el reconocimiento de Cantor en torno a los fundamentos del análisis.

¿Que pasaba cuando nuestro matemático comenzaba a tener un reconocimiento?, volvamos al designio paterno, al destino inscrito en esta carta, Georg ya empezaba a ser más importante que Theodore Schaeffer, pero también era cierto que comenzaría una persecución de aquellos colegas que alguna vez ocuparon el lugar de padres teóricos; la interlocución académica se convirtió en 


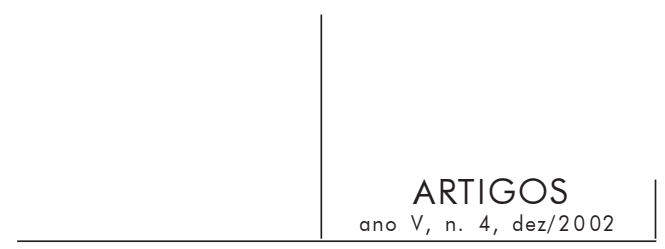

un lugar de progresiva alucinación psicótica, en este sentido, el desarrollo matemático y el reconocimiento se convirtieron en un lugar de desubjetivación.

Podemos ver algunos otros casos de la historia, donde la psicosis contribuyó a la realización de teorías revolucionarias, por ejemplo, Raimundo Lulio creó algunos de los fundamentos de las matemáticas que ahora utilizamos en la computación, asimismo creó una máquina delirante para corregir errores a través de la cual hablaba Dios. Cantor se funda en Leibnitz para desarrollar la extensión de su teoría hacia una cosmovisión fundada en las monadas a través del estudio de los transfinitos. Pascal también desarrolló esta misma línea de matemáticas con una fuerte creencia religiosa; hasta Cantor, la historia de las matemáticas iba directamente relacionada a la visión de Dios, todos estos son diferentes discursos de la eternidad, otro de estos es el delirio místico de los santos, estas figuras míticas son también modos de representar lo eterno e ilimitado.

\section{La constelación persecutoria}

Mientras ganaba terreno la teoría, las respuestas de los colegas disidentes aumentaban de volumen y cantidad, la teoría de Cantor representaba definitivamente una de las revoluciones epistemológicas más importantes de la historia, la amenaza de la destitución siempre estaba ahí, tal como lo decía la carta del padre; la potencia de Dios tenía siempre un lugar privilegiado. Schreber ${ }^{2}$ y Cantor se vivían como teniendo una relación privilegiada con Dios y, debido al contexto epistemológico, la polémica se vivía como una lucha entre la herejía y la deidad; la teoría de los transfinitos pulverizó esta noción y abrió el campo para una nueva concepción organizada y no teológica de lo eterno, esto se convierte en una trasgresión científica.

En la evolución psicopatológica de Georg Cantor, el lugar del padre se sustituyo por el lugar del perseguidor. En la instalación del delirio, los perseguidores son los maestros alemanes, quienes conspiraban contra él desde algunas sectas alemanas e inglesas que tenderían a quitarle un poder ilimitado.

En el siglo XIX el asunto de lo infinito es crucial en la polémica sobre las matemáticas, para Kroenecker, todo argumento filosófico y metodológico respecto al calculo del infinito, carece de sentido. En Kroenecker la aritmética podría abrir el camino a la geometría y al análisis.

Kroenecker en un principio fue un aliado, cuando impartió su seminario se convertiría en un disidente de la tradición académica presidida por Cantor; es

2. Caso estudiado por Freud. 


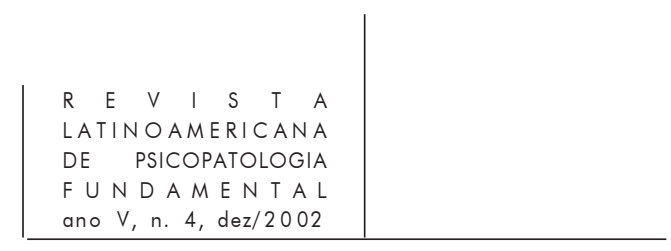

importante señalar que desde el lugar del saber institucional de la universidad, se realizaba una lucha de un paradigma contra otro, también Cantor amenazaba a sus contemporáneos.

Kroenecker lo veía como un tipo peligroso de matemática loca, las matemáticas se volverían una locura con Cantor; Kroenecker era apasionado de lo que llamaba la verdad de las matemáticas, él atacaba la teoría positiva del infinito tanto como a su autor, lo cual, finalmente lo llevó al hospital psiquiátrico, la locura fue un argumento recurrente contra su teoría.

En la primavera de 1884, a los 40 años, fue el primero de una serie de internamientos, una depresión profunda que lo llevaba a dudar de su teoría; alguna vez pidió cambiar de profesor de matemáticas al de filosofía en la Universidad de Halle; Kroenecker sacó a Cantor de la universidad de Berlín en 1884, este seminario cantoriano, se convierte para Kroenecker una herejía a combatir, en el cual se pone en juego el estatuto del infinito en matemáticas.

Cantor conoció a Dedekind en su luna de miel en 1874 en Intercalen, Dedekind simpatizó con Cantor como sujeto y con sus desarrollos teóricos. La alianza se produjo, pues cada uno de ellos desarrollaba modelos que venían a ser la disidencia del pensamiento tradicional; en síntesis, Dedekind es el primero en señalar que los números reales son de un numero mayor que los enteros, y los racionales mayor que los enteros; Dedekind y Cantor desarrollaban teorías similares en torno a la continuidad; otro de los interlocutores fue Weierstrass, quien formuló los principios de base del análisis en términos de límites, sin buscar evitar la presencia de series infinitas, por su parte Kroenecker seguía en las reglas finitistas.

Dedekind fungía como un padre que estimulaba las iniciativas de Georg, en este sentido podemos pensar que este acogimiento le permitió a Cantor desarrollar teorías desde un nicho teórico; este tipo de alianzas abrían el camino a la posibilidad de tener un lugar académico, formando así una relación epistemológica, a la manera de Sigmund Freud y Wilhelm Fliess.

\section{La destitución subjetiva: la caída en lo eterno}

Ser padre y publicar coinciden en el inicio de la caída. A los 29 años se casó con Vally Gutmann, con quien tuvo 2 hombres y 4 mujeres, este mismo año, comenzó a publicar; todo lugar que lo instituyera como un padre comenzaba a destituirlo como sujeto; el lugar de padre era imposible en Cantor, el Nombredel-Padre estaba ausente y a partir del espacio social que abría la teoría, comenzaba la pérdida del lazo social. 


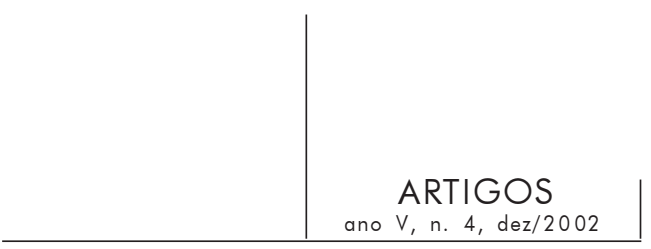

En 1884 después del primer conflicto con Mittag-Leffer, Cantor se refugio en la teología, su teoría empezó a despertar interés en las altas esferas del Vaticano. Después de la encíclica Aeterna patris pronunciada por el Papa Leo XIII en 1879, fue permitido la discusión de lo eterno en dios a partir de una idea tomista. Él aplico el pensamiento de los transfinitos a describir algunas de las formas de lo infinito, dios y del mundo. Lo cual fundamentaba la búsqueda epistemológica de la Iglesia frente a los nuevos tiempos.

La relación que Cantor guarda con el saber tiene una importancia crucial en su condición subjetiva. La producción de la teoría de los transfinitos daba a Cantor un modo de realizar nuevas aportaciones a la filosofía, la teología y la físico matemáticas. La reconstrucción de la idea de lo infinito tuvo necesariamente efectos en los modos de explicar científicamente la materia y la continuidad entre otros. En el transcurso de la vida de Cantor encontramos momentos muy precisos en esta producción estrechamente relacionados con los sucesos de su vida.

Sostener una teoría es una de las maneras de inscribirse en lo social. Kroenecker llamaba a la matemática de Cantor como una "matemática loca". Los periodos de internamiento le permitían desarrollar más sus propias construcciones. La producción de la ciencia siempre esta regida por el aparato psíquico. La escritura, la lógica que produce cada discurso es un representante del sujeto. La psicosis que sufría era, desde su propia lógica, una forma de pensar lo ilimitado. Este proceso resulta en una estructura particular del aparato psíquico constituida en otras leyes un estatuto distinto de lo inconsciente; además, imposibilidad de acceso a los simbólicos. El momento de aparición de las construcciones teóricas muestran de esta forma, la lógica de un sujeto, su autor; pero además pone en evidencia que el delirio es una de las formas epistemicas capaz de producir la ciencia desde su forma más pura.

La fantasmagoría comenzaba a tener un carácter de persecución, en 1884, el factor detonador de la primera serie de persecuciones, fue el recibimiento elogioso por parte de los matemáticos franceses que instituían a Cantor en una posición paternal: padre de una teoría y desde el lugar del maestro.

\section{Algunos modos de la eternidad: delirio y transfinitos}

Las hospitalizaciones tuvieron una función importante en el pensamiento cantoriano. En 1905, Cantor sale de la clínica de Halle. La contención hospitalaria le permitía elaborar campos epistémicos nuevos. Precisamente entre los episodios delirantes podría realizar nuevas aportaciones a su teoría. En estos episodios Dios 


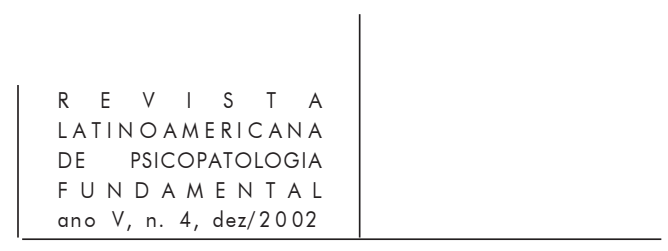

se convertía en un interlocutor imaginario. Dios se comunicaba con Cantor a través de revelaciones. Dios era el inventor de la teoría y Cantor era el escriba. Esta formula es especialmente interesante si precisamente en la psicosis muestra una desubjetivación por la disolución de las coordenadas de la subjetividad. Dios se impone, traiciona. Es el amo. En una de las pocas biografías sobre su vida, Dauven (1979) escribe:

También es significativo que Cantor creyera en la verdad absoluta de su teoría de los conjuntos porque había sido revelada por Dios para él. Quizá el se vía a si mismo no solo como el mensajero de Dios, registrando con precisión, reportando y transmitiendo la nueva teoría revelada de los números transfinitos sino además como el embajador de Dios. (p. 147)

Partamos de algunas hipótesis que dan título a este escrito, si sabemos que el delirio esta compuesto por puro proceso primario, entonces cabe preguntarnos cuál es su temporalidad; como señala Freud y Lacan el campo de lo ilimitado, el Otro como universo de significantes, es un modo de lo eterno.

Es también pertinente señalar que la lógica de la psicosis opera a partir de la forclusión, desde ahí no hay espacio para la duda, la certeza entonces cobra un especial contexto en este campo. En matemáticas se juegan verdades lógicas, no hay espacio para la duda, es el campo de la proposición pura, terreno fácil para la certeza: modo lógico de construcción del delirio. Cantor escribe:

Mi teoría es fuerte como una piedra; toda flecha dirigida contra ella será rápidamente regresada contra su arquero. ¿Cómo es que yo se esto?, porque yo la he estudiado sobre todos sus ángulos durante años, porque yo examiné todas las objeciones que fueron hechas contra el infinito, y sobre todo, porque yo he seguido sus raíces, si yo lo puedo decir, en la causa primera infalible de todas las cosas creadas". (Charraud, 1994 - Traducción del autor)

¿Qué parte de su teoría era delirio y cual de ella era precisamente una verdad lógica incuestionable?, su delirio también tendería a organizar una serie de significantes de una manera particular, las relaciones que establece entre su descubrimiento y su delirio siempre son consistentes, en Cantor, la relación que guarda su delirio con su teoría es definitiva.

\section{La inducción matemática y el delirio}

Aquí es preciso traer a cuenta a la inducción matemática. Lo representable es un fenómeno de las construcciones científicas. La formulación de las teorías pasa desde los sueños hasta el delirio. No hay producción sin sujeto: desde ahí 


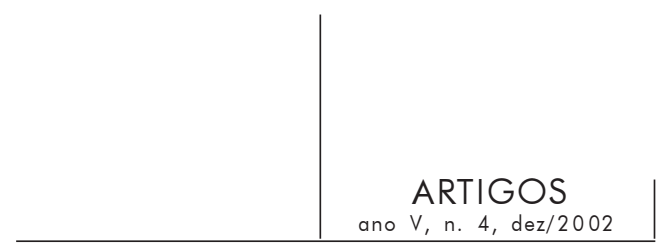

cabe preguntarnos. ¿Qué es la inducción matemática desde el psicoanálisis? Primero en la invención científica y después desde la lógica de la psicosis. La propuesta de los transfinitos necesariamente se sitúa entre estas fronteras. El lenguaje como estructura deviene un corpus de signos. Lógica inequívoca y de certezas. El mundo de las matemáticas no es el del símbolo, sino el del signo. Lacan (1984) escribe en su seminario sobre las psicosis:

Las matemáticas en cambio, utilizan un lenguaje de puro significante, un metalenguaje por excelencia. Reducen al lenguaje a su función sistemática sobre la cual esta construido otro sistema de lenguaje, incluyendo al primero en su articulación. La eficacia de este modo de proceder no es dudosa en su registro propio. (p. 132)

Las matemáticas como contexto epistémico son un "lenguaje de puro significante" pero esto no resuelve la relación entre el delirio y la inducción matemática. La lógica del delirio también abría un campo para pensar lo ilimitado. Solo a partir del autor podemos abarcar su producción. La lógica del delirio entonces producía la teoría de los transfinitos. La inducción matemática era revelada por Dios. Produciendo así una escritura posible de la psicosis. Charraud (1994) sostiene que: "En la teoría de los transfinitos y en la construcción de los números reales encontramos el fantasma del cuerpo fragmentado" (p. 236).

La idea de Cantor sobre la inducción muestra su relación con las matemáticas. Además la postura que guarda el mundo material con respecto a las matemáticas. La idea de que las matemáticas eran del orden de la metafísica; liberaba a la abstracción a sus propios caminos. Vayamos a sus propias palabras:

La esencia de las matemáticas es su libertad. Por su posición extraordinaria que distingue a las matemáticas de todas las ciencias, y la cual produce una explicación para la relativamente libre y fácil manera de alcanzarlo, merece especialmente el nombre de matemáticas libres, un nombre que yo, si tuviera la oportunidad, preferiría al actual de matemáticas puras. (Dauven, 1979, p. 132)

Las matemáticas puras, libres, pero al mismo tiempo desubjetivadas; la idea delirante de ser el escriba de Dios muestra una relación imaginaria con dios. Cantor solo escribía lo que Dios le dictaba. Dios ubicado como absoluto. Ilimitado. Infinito. Finalmente el lugar del padre de los transfinitos, Cantor lo proponía en Dios. Cantor mismo, igual que su padre en su carta eran solo participes del deseo de Dios. Un sujeto imposible, una caída en lo eterno. 


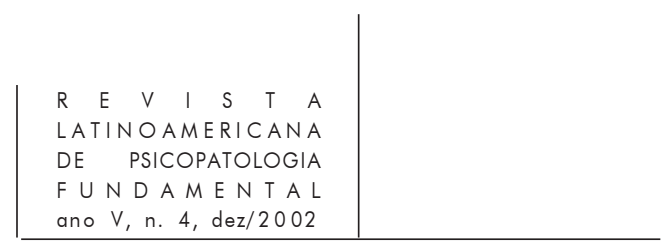

\section{Referencias}

Bell, E. Men of mathematics. New York: Dover, 1937.

Charraud, N. Infini et Inconscien: Essay sur Georg Cantor. Paris: Anthropos, 1994.

DAUVEN, J. Georg Cantor: his mathematics and filosophy of the infinite. New Jersey:

Princeton, 1979.

LACAN, J. El seminario. Libro 3. Las psicosis. Buenos Aires: Paidós, 1955.

El seminario. Libro 11. Los cuatro conceptos fundamentales del psicoanálisis.

Buenos Aires: Paidós, 1984.

\section{Resumos}

Este artigo coloca em discussão a relação entre estrutura psíquica e a produção lógica do delírio, partindo de um matemático alemão chamado Georg Cantor. Discutese ainda a relação entre a produção matemática e a instalação de sua psicose.

Palavras-chave: Infinitos, psicose, matemáticas, conjuntos

Cet article met en discussion la relation entre la structure psychique et la production logique du délire à partir du mathématicien allemand Georg Cantor. La relation entre la production mathématique et l'installation de la psychose est également discuté ici.

Mots clés: Infini, psychose, mathématiques, ensembles

This article discusses the relationship between psychological structure and the logical production of delirium, based on the case of the German mathematician Georg Cantor. The relationship between mathematical production and the installation of his psychosis are also discussed.

Key words: Infinite, psychosis, mathematics, sets

Versão inicial recebida em outubro de 2001

Aprovado para publicação em novembro de 2002 